\title{
Foreword
}

\section{Electrocardiographic Trilogy}

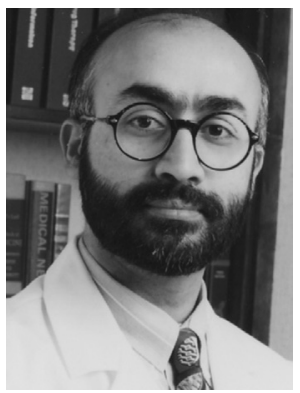

Ranjan K. Thakur, MD, MPH, MBA, FHRS

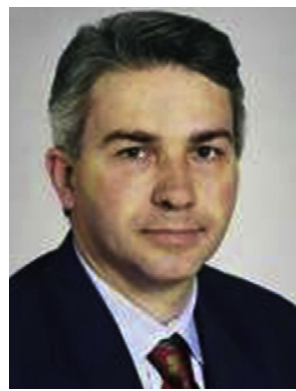

Andrea Natale, MD, FACC, FHRS

Consulting Editors

We are pleased to introduce this issue of Cardiac Electrophysiology Clinics devoted to interpretation of complex arrhythmias. This is the third issue of a trilogy on interpretation of the 12-lead electrocardiogram (ECG). Dr Luigi Padeletti started this project, but he passed away unexpectedly while working on the second issue. We appreciate and congratulate his colleagues, Drs Giuseppe Bagliani, Roberto De Ponti, and Fabio Leonelli, for bringing Luigi's dream to fruition by completing the trilogy.

The first issue in this series (September 2017, volume 9 , issue 3 ) focused on basic electrocardiography in normal and diseased hearts. It took a unique approach to discussing electrocardiography of arrhythmias. It first detailed what can be learned about physiology, pathology, and neural control from each wave and interval of the ECG and then built on that to discuss arrhythmias originating in each cardiac structure. The second issue (June 2018, volume 10, issue 2) focused on electrocardiography of various arrhythmias: bradycardias, tachycardias, and some specific arrhythmias. This issue focuses on interpretation of complex arrhythmias due to problematic ECGs or intricate arrhythmia mechanisms.

Again, we congratulate the editors for fulfilling the vision of one of their colleagues. It speaks to their love and admiration for Dr Luigi Padeletti. We hope the readers will dust off the previous issues in this trilogy and enjoy reading all three volumes together.

Ranjan K. Thakur, MD, MPH, MBA, FHRS Sparrow Thoracic and Cardiovascular Institute Michigan State University 1200 East Michigan Avenue, Suite 580 Lansing, MI 48912, USA

Andrea Natale, MD, FACC, FHRS Texas Cardiac Arrhythmia Institute

Center for Atrial Fibrillation at

St. David's Medical Center 1015 East 32nd Street, Suite 516 Austin, TX 78705, USA

E-mail addresses:

thakur@msu.edu (R.K. Thakur) andrea.natale@stdavids.com (A. Natale) 